\title{
Haemostatic variables associated with diabetes and its complications
}

\author{
J H FULLER, H KEEN, R J JARRETT, T OMER, T W MEADE, R CHAKRABARTI, \\ W R S NORTH, YVONNE STIRLING
}

thrombogenic component in the vascular complications of diabetes would suggest that prevention and treatment of these complications should include antithrombotic agents.

Studies on haemostatic function in diabetes ${ }^{4-8}$ have mostly

\section{Summary and conclusions}

To study the possible role of an "increased thrombotic tendency" in the vascular complications of diabetes several tests of haemostatic function were carried out on 91 men and 63 women with diabetes aged 35-54 years and the results compared with findings in 686 men and 393 women of the same age in the Northwick Park Heart Study. Mean values for factors VII and X, fibrinogen, and platelet adhesiveness were higher in the diabetics, but mean fibrinolytic activity and whole blood platelet counts were lower. Antithrombin III values were also higher in the diabetics, which may have constituted a protective response to other changes favouring the onset of vascular disease. Diabetics with retinopathy had higher factor VII and antithrombin III values, and those with proteinuria had higher values for factor VII, fibrinogen, and platelet adhesiveness than those without these complications.

These findings suggest a potentially important association between a thrombogenic tendency and vascular disease in diabetes. Nevertheless, prospective data are needed to clarify whether the haemostatic abnormalities precede the onset of clinically manifest vascular complications or are a consequence of them.

\section{Introduction}

The excess cardiovascular morbidity and mortality among diabetics in developed countries have not been fully explained by major risk factors such as hypertension, cigarette smoking, and hypercholesterolaemia. ${ }^{1}$ Increasing attention is being paid to disordered haemostatic mechanisms in the pathogenesis of vascular disease in non-diabetics, ${ }^{2}$ and such disorders may be of special importance in determining the increased risk of both large-vessel and small-vessel disease in diabetes. ${ }^{3}$

Evidence of an "increased thrombotic tendency" in diabetes would have aetiological, preventive, and therapeutic implications. Findings in a high-risk group such as diabetics may be relevant not only to diabetes itself but also to the pathogenesis of arterial disease in general. Furthermore, strong evidence for a

\footnotetext{
Department of Medical Statistics and Epidemiology, London School of Hygiene and Tropical Medicine, London WC1 7HT

J H FULLER, MRCP, senior research fellow

Guy's Hospital, London SE1 9RT

H KEEN, MD, FRCP, professor of human metabolism

R J JARRETT, MD, MFCM, senior lecturer in community medicine

$T$ OMER, BSC, research associate, unit for metabolic medicine

MRC/DHSS Epidemiology and Medical Care Unit, Northwick Park Hospital, Middlesex HA1 3UJ

$T$ W MEADE, FRCP, FFCM, director

R CHAKRABARTI, MB, MRCPATH, member of scientific staff

W R S NORTH, MA, MSC, member of scientific staff

YVONNE STIRLING, FIMLS, chief technician
}

been small, and in none has a wide range of tests of haemostatic function been applied to groups large enough to see whether, for example, patients with insulin-dependent diabetes differ from those with non-insulin-dependent diabetes. As part of a multinational study of vascular disease in diabetes sponsored by the World Health Organisation and the International Diabetes Federation several haemostatic variables have been measured in 154 diabetics and the findings compared with those in 1079 age-matched participants in the Northwick Park Heart Study. ${ }^{9}$

\section{Subjects and methods}

The 154 diabetics were selected at random from the registers of two London diabetic clinics. Their ages ranged from 35 to 54 years, and all had been born in the British Isles. Of the 91 men and 63 women in the sample, 61 and 50 respectively had insulin-dependent diabetes (IDD). Of the 30 men with non-insulin-dependent diabetes (NIDD), 12 were receiving sulphonylureas, 2 biguanides, 6 both agents, and 10 dietary management alone. Of the 13 women with NIDD, 4 were receiving sulphonylureas, 1 biguanides, 4 both agents, and 4 diet alone. Responses to the WHO cardiovascular questionnaire $^{10}$ were recorded and a 12-lead resting electrocardiogram (ECG) coded independently according to the Minnesota classification by two trained observers.

Funduscopy was carried out under standard conditions by two experienced clinicians. Each eye was viewed for 120 seconds with a direct ophthalmoscope in a darkened room after dilatation of the pupils $(3 \mathrm{~mm})$. Retinopathy was recorded if red lesions (haemorrhages or microaneurysms), hard or soft exudates, or new vessel systems were noted in either eye. Retinopathy was present in 42 men and 25 women. Proteinuria was sought by the salicylsulphonic-acid test on an early-morning sample, any degree of turbidity being recorded as positive. Seventeen men and eight women had proteinuria. Altogether 79 of the 154 patients $\left(51^{\circ}\right)$ ) had either retinopathy or proteinuria.

Findings in the diabetics were compared with those in 686 white men and 393 white women aged 35-54 years recruited into the Northwick Park Heart Study (NPHS) over the same period. Women taking oral contraceptives were excluded. Although six diabetics (five men and one woman) in the NPHS group are included in the analyses, the group as a whole is referred to as non-diabetic. The NPHS is a prospective survey of ischaemic heart disease in several occupational groups in north-west London; further details, including laboratory methods, are given eisewhere. ${ }^{911} 12$ The haemostatic variables measured in the diabetic and non-diabetic groups were factors V, VII, and VIII; fibrinogen concentration; fibrinolytic activity (defined as 100 /ysis time in hours); antithrombin III (biological); platelet count; and platelet adhesiveness. Factors II and X were assayed in a subgroup of 33 men with IDD and the results compared with findings in 19 non-diabetic men of similar age, whose samples were assayed at the same time and against the same standard.

In both the diabetic and non-diabetic groups blood was taken without venecompression (unless otherwise unobtainable) between 0700 and 1100. At the time of sampling, the diabetics had been fasting for 12 hours and had not had their morning dose of insulin or oral treatment. Blood samples from the non-diabetics, however, were taken after their usual breakfast. Multiple regression analysis in the NPHS sample showed no association between diet after rising (total energy or proportion of energy derived from carbohydrate, 
fat, or protein) and any of the haemostatic variables examined, with the exception of an apparent lowering of platelet adhesiveness by about $9{ }_{0}^{\circ} / 1000 \mathrm{kcal}(4 \cdot 2 \mathrm{MJ})$. The average breakfast provided about $300 \mathrm{kcal}$, so that the non-fasting values for platelet adhesiveness in this group may have been about $3^{\prime \prime}$ "lower on average than if samples had been taken during fasting. With this possible exception the difference in recent food intake between the two groups seemed unlikely to account for any haemostatic differences observed.

Since age and the duration of diabetes are associated with each other and with the development of arterial disease, retinopathy, and values were also higher in the diabetics, the differences achieving significance for patients with IDD and all diabetics combined.

Fibrinolytic activity was highly significantly less in men and women with NIDD than in non-diabetics. From multiple regression analysis this difference was to only a small extent attributable to the greater obesity of patients with NIDD. Fibrinolytic activity was also less in women with IDD than in non-diabetics.

Platelet adhesiveness was consistently and substantially greater in the diabetics than non-diabetics; platelet count was consistently lower.

Assay of factors II and X in 33 men with IDD and 19 non-diabetic

TABLE II-Haemostatic variables in diabetic and non-diabetic (NPHS participants) groups. Values are means $\pm S D$

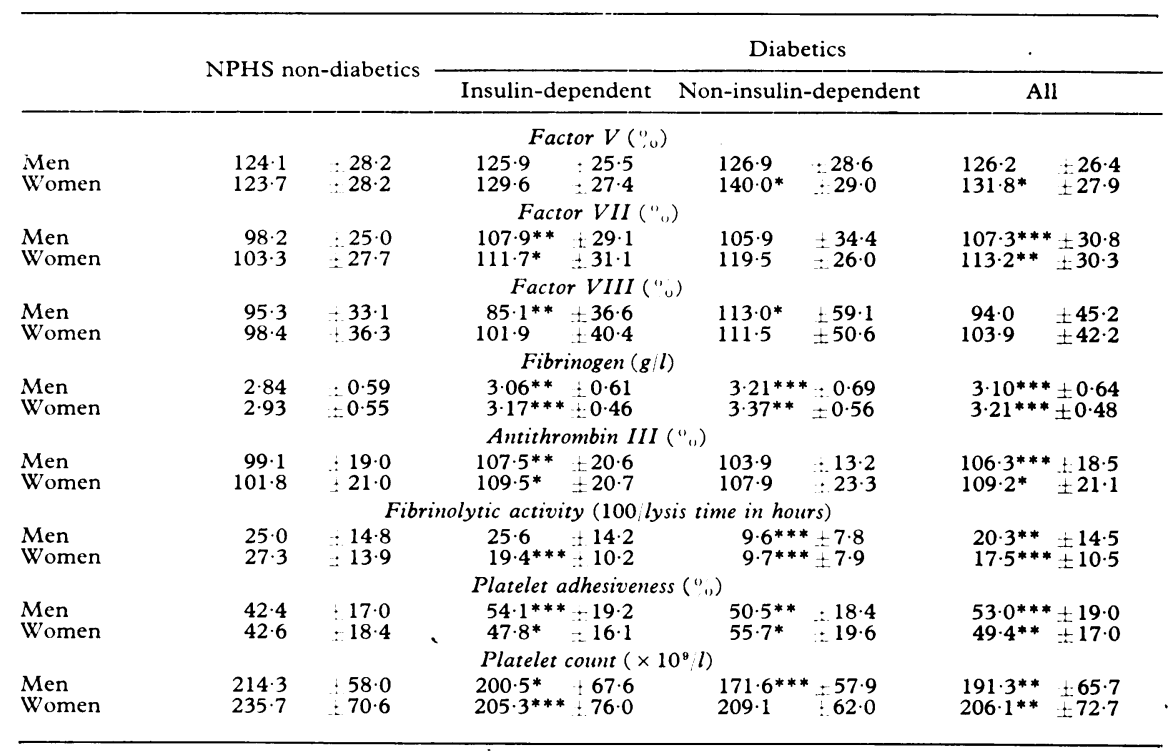

Diabetics $v$ non-diabetics: $\mathrm{P}^{*}<0.05 ;{ }^{* *} \mathrm{P}<0.01 ; * * * \mathrm{P}<0.01$.

proteinuria they were taken into account when analysing the relation of the haemostatic variables to these complications by using stepwise multiple regression. The presence or absence of the complication (retinopathy or proteinuria) was taken as the dependent variable and age, sex, duration of diabetes, insulin dependence or otherwise, smoking, blood pressure, and the eight measures of haemostatic function as the independent variables.

\section{Results}

Table Im (miniprint) gives details of age, adiposity, and cigarette smoking and the prevalence of ischaemic heart disease in the diabetic and non-diabetic groups. Patients with IDD were slightly younger on average than those with NIDD. Compared with the non-diabetics fewer patients with IDD but more with NIDD were cigarette smokers. Mean body-mass index (weight $(\mathrm{kg}) /$ height* $(\mathrm{m})$ ) was lower in the IDD group but higher in the NIDD group than in the nondiabetics. The prevalence of clinical or electrocardiographic evidence of ischaemic heart disease was considerably greater in both diabetic groups than in the non-diabetics.

Table II lists the haemostatic values in the diabetic and nondiabetic groups. The mean factor $\mathrm{V}$ value was significantly higher than in non-diabetics only in women with NIDD. Factor VII values, however, were consistently higher in the diabetics than non-diabetics, and significantly so in men and women with IDD. Table IIIm amplifies this difference, giving the distribution of factor VII values in diabetics according to the low, middle, and high thirds of their distribution in the non-diabetics.

There was an inconsistent pattern for factor VIII. Men with IDD had a significantly lower factor VIII value than the non-diabetics, but the reverse was true for men with NIDD; when all the diabetics were considered together there was no difference. There was no significant difference in factor VIII values between diabetic and nondiabetic women.

Fibrinogen concentrations were consistently higher in both the IDD and NIDD groups than in non-diabetics. Antithrombin III men showed a significantly higher mean factor $\mathrm{X}$ value in the diabetics. Factor II values, however, did not differ significantly (table IVm).

Table $\mathrm{V}$ gives the findings for all diabetics according to the presence and absence of retinopathy and proteinuria. Factor VII and antithrombin III values were significantly higher in those with retinopathy than in those without. Factor VII values, fibrinogen concentrations, and degree of platelet adhesiveness were all higher in those with proteinuria than in those without. Patients with "complicated"

MINIPRINT TABLES

Im

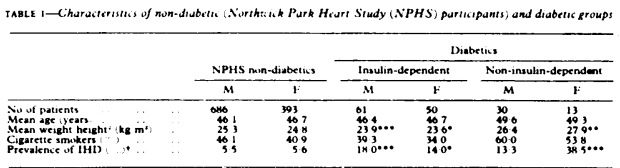

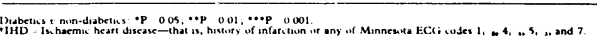

IIIm

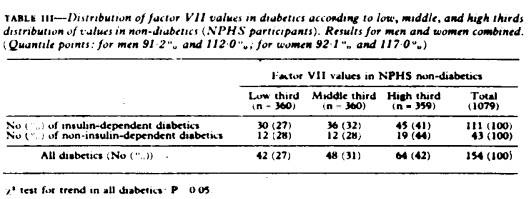

$\mathrm{IVm}$

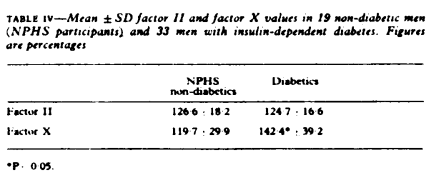


disease-that is, with either retinopathy or proteinuria or bothhad significantly higher values for factors V and VII, fibrinogen, and antithrombin III than those with "uncomplicated" disease. Multiple regression analysis showed that the associations of antithrombin III with retinopathy and of factor VII, fibrinogen, and platelet adhesiveness with proteinuria occurred independently of age, sex, duration and type of diabetes, smoking, and blood pressure.

There was no difference in any haemostatic variable between diabetics with and without clinical or electrocardiographic evidence of ischaemic heart disease. antithrombin III (which may also indicate a protective response), fibrinogen, and platelet adhesiveness suggest a role for these variables in microvascular disease but were not entirely consistent in the retinopathy and proteinuria groups. In contrast to Almér et al, ${ }^{21}$ we did not find impaired fibrinolytic activity in diabetics with retinopathy compared with those without.

In summary the results of this cross-sectional study confirm that diabetics have abnormalities of coagulation factors, fibrino-

TABLE V-Haemostatic variables in diabetics with and without retinopathy and proteinuria. Male and female insulin-dependent and non-insulin-dependent groups combined. Values are means

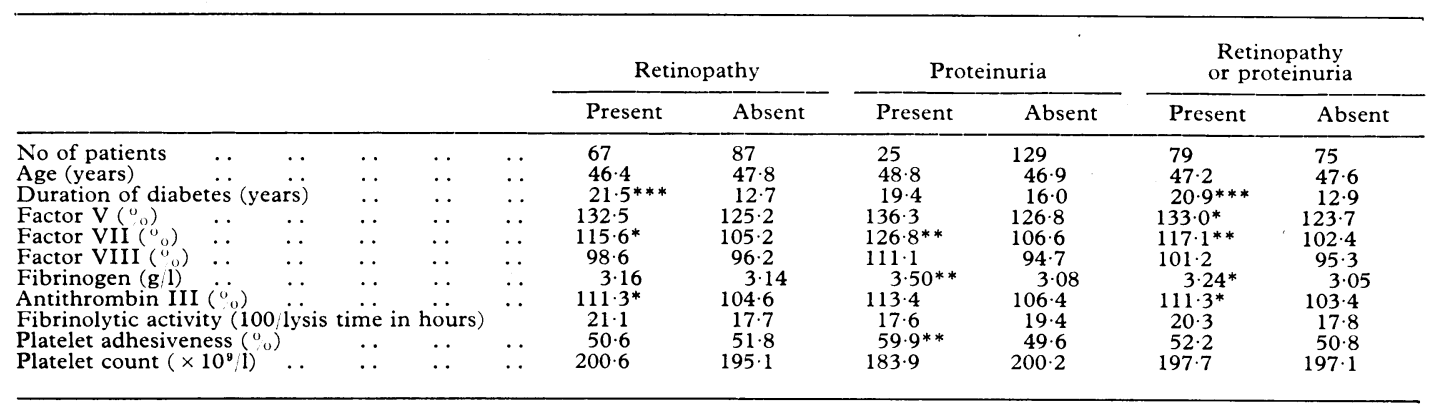

Diabetics with complications $v$ those without: $* \mathbf{P}<0.05 ; * * \mathbf{P}<0.01 ; * * * \mathbf{P}<0.001$.

\section{Discussion}

In comparing the diabetics with non-diabetics (tables II and $\mathrm{IVm}$ ) the findings for factors VII and X, fibrinogen, fibrinolytic activity, and platelet adhesiveness were all compatible with the hypothesis that disordered haemostatic function favouring an increased thrombotic tendency is associated with the specific vascular complications of diabetes. Our results, in large groups of non-diabetic and diabetic subjects, broadly agreed with those of other workers. ${ }^{5} 61314$ The lower platelet count in the diabetics may have been due to an increased platelet turnover as part of a generally increased level of platelet activity..$^{15}$ Even allowing for the possibility that recent food intake lowers platelet adhesiveness to the extent suggested by the NPHS data, the difference between the non-diabetics and diabetics was substantial, particularly in men (table II).

Some groups of diabetics have increased sensitivity to plateletaggregating agents ${ }^{16}$ which may be associated with increased platelet prostaglandin synthesis or an interaction with plasma factors, such as the von Willebrand factor. ${ }^{17}$

Our results for factor VIII were inconsistent and do not confirm the reported difference between non-diabetics and diabetics. ${ }^{4}$

The poorer fibrinolytic activity in the diabetics was mainly attributable to the NIDD group. This impaired activity was probably not due to oral antidiabetic agents, since Bogie et al ${ }^{18}$ found no significant change in fibrinolytic activity over one year in patients with NIDD allocated at random to treatment with glibenclamide, phenformin, both drugs, or diet alone.

At first sight the direction of difference in antithrombin III values between the non-diabetics and diabetics seems paradoxical; diabetics might be expected to have lower values in view of the increased risk of thromboembolic disease in those with congenital antithrombin III deficiency. ${ }^{19}$ Patients without congenital deficiency who have had clinical episodes of ischaemic heart disease or are at risk of developing the disease, however, have high antithrombin III values,,$^{20}$ possibly as a protective response. A similar explanation may apply in diabetes.

Our results also suggest that an increased thrombotic tendency may be related to the onset of microvascular disease in diabetes, although as with the findings already discussed prospective data are needed to distinguish cause and consequence. Mean factor VII values were higher in diabetics with retinopathy and proteinuria than in those without (table V). The findings for lytic activity, and platelet function. This "increased thrombotic tendency" may play a part in the vascular complications of diabetes. Studies are needed to show whether correction of these haemostatic abnormalities by therapeutic intervention or improved diabetic control or both will lower the incidence of large-vessel and small-vessel diseàse in diabetes.

We thank the collaborating physicians at the London centre of the Multinational Study of Vascular Disease in Diabetes-namely, Drs D A Pyke, P J Watkins, R Smith, G C Viberti and S M Bakirand the diabetic patients at King's College and Guy's hospitals, London, who took part in the study. H J Heinz Co Ltd, the London Borough of Harrow and Brent, and the north-west London telephone area of the Post Office are thanked for their participation in the Northwick Park Heart Study. This study was supported by grants from the Medical Research Council and the British Diabetic Association.

Requests for reprints should be addressed to: Dr J H Fuller, Department of Medical Statistics and Epidemiology, London School of Hygiene and Tropical Medicine, London WCIE 7HT.

\section{References}

${ }^{1}$ Fuller, J H, et al, fournal of Chronic Diseases. In press.

2 Mustard, J F, Packham, M A, and Kinlough-Rathbone, R L, Advances in Experimental Medicine and Biology, 1978, 102, 7.

3 McMillan, D E, Diabetes, 1975, 24, 944.

${ }^{4}$ Egeberg, O, Scandinavian fournal of Clinical and Laboratory Investigation, 1963, 15, 533.

${ }^{5}$ Fearnley, G R, Chakrabarti, R, and Avis, P R D, British Medical fournal, 1963, 1, 921.

${ }^{6}$ Mayne, E E, Bridges, J M, and Weaver, J A, Diabetologia, 1970, 6, 436.

' Heath, H, et al, Diabetologia, 1971, 7, 308.

${ }^{8}$ O'Malley, B C, et al, Lancet, 1975, 2, 1274.

${ }^{9}$ Meade, T W, and North, W R S, British Medical Bulletin, 1977, 33, 283.

${ }^{10}$ Rose, G A, and Blackburn, H, World Health Organisation Monograph Series, 1968, No 56, p 172.

${ }^{11}$ Meade, T W, et al, British fournal of Haematology, 1976, 34, 351.

${ }^{12}$ Meade, T W, et al, Lancet, 1977, 2, 948.

13 Valdorf-Hansen, F, Danish Medical Bulletin, 1967, 14, 244.

${ }_{14}^{14}$ Hellem, A J, Acta Medica Scandinavica, 1971, 190, 291.

${ }^{15}$ Ferguson, J C, et al, Clinical Science and Molecular Medicine, 1975, 49, 115.

${ }^{16}$ Bern, M M, Diabetes, 1978, 27, 342.

17 Colwell, J A, et al, Advances in Experimental Medicine and Biology, 1978, $102,243$.

${ }_{18}$ Bogie, W, George, J, and Crane, C W, Lancet, 1976, 2, 312.

19 Mackie, M, et al, British Medical fournal, 1978, 1, 136.

20 Yue, R H, et al, Thrombosis and Haemostasis, 1976, 35, 598.

${ }^{21}$ Almér, L-O, Pandolfi, M, and Nilsson, I M, Diabetes, 1975, 24, 529. 